\title{
Beyond the androgen receptor II: New approaches to understanding and treating metastatic prostate cancer; Report from the 2017 Coffey-Holden Prostate Cancer Academy Meeting
}

\author{
Andrea K. Miyahira ${ }^{1}$ (iD \\ Heather H. Cheng ${ }^{2,3}$ | Wassim Abida ${ }^{4}$ | Leigh Ellis ${ }^{5,6}$ \\ Lauren C. Harshman ${ }^{7}$ (iD \\ Daniel E. Spratt $^{8}$ | Jonathan W. Simons ${ }^{1}$ । \\ Kenneth J. Pienta9,10,11 \\ Howard R. Soule ${ }^{1}$
}

1 Prostate Cancer Foundation, Santa Monica, California

${ }^{2}$ Department of Medicine, University of Washington, Seattle, Washington

${ }^{3}$ Division of Clinical Research, Fred Hutchinson Cancer Research Center, Seattle, Washington

${ }^{4}$ Genitourinary Oncology Service, Department of Medicine, Memorial Sloan Kettering Cancer Center, New York, New York

${ }^{5}$ Department of Oncologic Pathology, Dana-Farber Cancer Institute, Boston,

Massachusetts

${ }^{6}$ Department of Pathology, Brigham and Women's Hospital and Harvard Medical School, Boston, Massachusetts

${ }^{7}$ Lank Center for Genitourinary Oncology, Dana-Farber Cancer Institute and Harvard Medical School, Boston, Massachusetts

${ }^{8}$ Department of Radiation Oncology, University of Michigan, Ann Arbor, Michigan

${ }^{9}$ Department of Oncology, Sidney Kimmel Comprehensive Cancer Center, The Johns Hopkins School of Medicine, Baltimore, Maryland

10 Department of Urology, The James Buchanan Brady Urological Institute, Baltimore, Maryland

11 Department of Pharmacology and Molecular Sciences, The Johns Hopkins School of Medicine, Baltimore, Maryland

\section{Correspondence}

Howard R. Soule, Prostate Cancer Foundation, 1250 4th Street, Santa Monica, CA, 90401.

Email: hsoule@pcf.org

Introduction: The 2017 Coffey-Holden Prostate Cancer Academy (CHPCA) Meeting, "Beyond the Androgen Receptor II: New Approaches to Understanding and Treating Metastatic Prostate Cancer," was held in Carlsbad, California from June 14-17, 2017. Methods: The CHPCA is an annual scientific conference hosted by the Prostate Cancer Foundation (PCF) that is uniquely designed to produce extensive and constructive discussions on the most urgent and impactful topics concerning research into the biology and treatment of metastatic prostate cancer. The 2017 CHPCA Meeting was the 5 th meeting in this annual series and was attended by 71 investigators focused on prostate cancer and a variety of other fields including breast and ovarian cancer.

Results: The discussions at the meeting were concentrated on topics areas including: mechanisms and therapeutic approaches for molecular subclasses of castrate resistant prostate cancer (CRPC), the epigenetic landscape of prostate cancer, the role of DNA repair gene mutations, advancing the use of germline genetics in clinical practice, radionuclides for imaging and therapy, advances in molecular imaging, and therapeutic strategies for successful use of immunotherapy in advanced prostate cancer.

Discussion: This article reviews the presentations and discussions from the 2017 CHPCA Meeting in order to disseminate this knowledge and accelerate new biological understandings and advances in the treatment of patients with metastatic prostate cancer.

\section{KEYWORDS}

biomarkers, cancer immunotherapy, molecular imaging, therapeutics, tumor genomics 


\section{1 | INTRODUCTION}

The Prostate Cancer Foundation (PCF) has a legacy of driving new innovative solutions that have reduced death and suffering from prostate cancer through funding critical research, recruiting human capital, supporting young investigators, facilitating global knowledge exchange, driving new collaborations, influencing policy, and convening scientific conferences.

The Coffey-Holden Prostate Cancer Academy (CHPCA) Meeting is an annual conference convened by PCF that gathers $\sim 75$ investigators to discuss the most critical avenues of research that necessitate study in order to accelerate improved treatments for men with advanced prostate cancer. ${ }^{1-4}$ The CHPCA Meeting is uniquely structured into short talks followed by long discussions for the purpose of generating a "think tank" atmosphere that stimulates brainstorming and the development of strategic new ideas, projects, and collaborations in most needed areas of research. A second goal of this conference is to promote the development of early career investigators. Young investigators comprise the meeting organizing committee as well as roughly half of meeting attendees. This conference is modeled after the NCl's Prouts Neck Meetings on Prostate Cancer, which took place from 1985 through 2007.5 The CHPCA Meeting has been convened annually by PCF since 2013 , and is named for two individuals who made huge and invaluable contributions toward prostate cancer research and treatment, Dr. Donald Coffey and Dr. Stuart Holden.

The 2017 CHPCA Meeting took place from June 14-17, 2017, in Carlsbad, California, and was themed "Beyond the Androgen Receptor II: New Approaches to Understanding and Treating Metastatic Prostate Cancer." The meeting was attended by 71 investigators which included 41 PCF-funded young investigators. The meeting focused on several of the most promising avenues for improved biological understanding and treatment of metastatic prostate cancer. These included: biology and treatment approaches for aggressive variant castrate resistant prostate cancer (CRPC), epigenetic regulation of CRPC, the role of DNA repair gene mutations in driving prostate and other cancers, strategic approaches to delivering germline genetics into actionable clinical practice, development of targeted radionuclides for imaging and therapy, advances in molecular imaging, and therapeutic strategies for successful use of immunotherapy in advanced prostate cancer. In addition to prostate cancer, experts from several other major fields unrelated to prostate cancer research attended and contributed fresh research insights and lessons from breast cancer, ovarian cancer, and astronomy/high-dimension informatics that may be applied to improve studies in prostate cancer.

\subsection{The role of DNA repair in prostate cancer}

Studies into mechanisms of DNA repair in prostate cancer have gained new prominence owing to the recognition that approximately $25 \%$ of metastatic castration resistant prostate cancers carry defects in DNA repair, particularly in homologous recombination (e.g. BRCA2, ATM, BRCA1) and mismatch repair (e.g. MLH1, MSH2, MSH6, PMS2). ${ }^{6}$ Emerging data suggests prostate cancers with homologous recombination deficiency (HRD) may be sensitive to poly(ADP) ribosylase inhibitors (PARPi) and platinum chemotherapy. ${ }^{7,8}$ These represent potential net additions to the available treatment options for patients and clinical trials of PARPi in prostate cancer are currently underway (e.g. NCT02987543, NCT02952534, and NCT02975934).

Olaparib, rucaparib, and niraparib are now FDA-approved for ovarian cancer and it is worth considering the parallels with prostate cancer. BRCAmutated ovarian cancers have excellent responses to PARPi and the response rates appear similar between germline and somatic HRD. However, observed responses in the absence of BRCA mutations suggest other factors contribute to PARPi sensitivity. Reported mechanisms of HRD include mutations, alterations in gene expression, miRNA expression, epigenetic modification such as methylation, and/or changes in protein expression. Efforts are underway to characterize the effects of different alterations in HRD genes to identify biomarkers that can select patients most likely to respond to treatment with platinum and PARPi. Alternative approaches include identification of functional consequences of HRD such as genomic scarring, analysis of mutational spectra, and/or functional analyses, such as in cell models. Further efforts to characterize less commonly mutated genes such as CHEK2, BRIP1, CDK12, and RAD51C among others, as well as to explore epigenetic regulation of these genes with respect to HRD and to sensitivity to platinum and PARPi will be critical.

Unfortunately, while PARPi and platinum-based chemotherapy can lead to dramatic responses, resistance eventually develops and there is a need to better understand these mechanisms to develop strategies aimed at overcoming resistance. Secondary somatic mutations that restore function of genes initially inactivated have been described in ovarian cancer ${ }^{9-12}$ and have now been reported in prostate cancer. ${ }^{13,14}$ As molecular profiling methods mature, early detection of resistance may facilitate additional strategies to predict and enhance sensitivity and to prevent resistance.

SPOP mutations are commonly observed in primary and metastatic prostate cancer, ${ }^{6,15}$ and preclinical evidence suggests their role in directing the balance of homologous recombination and nonhomologous end joining DNA repair pathways. Initial data suggests that SPOP mutations are not sufficient to predict response to PARPi in CRPC and further work is needed to understand the biological importance of SPOP mutations and their interactions with key regulators such as androgen receptor (AR) and DNA repair in prostate cancer biology.

Ongoing work will identify alternative mechanisms of HRD orthologs in cancers without known HRD that may sensitize to PARPi or create synergy with radiation such as Lu177 (a beta-emitter) and Ac225 (an alpha-emitter). Further understanding of the molecular interactions between AR regulation and PARP activity, and upstream interactions such as HOXA9-TWIST interaction with PARPi may reveal additional new therapeutic strategies.

\section{2 | Advancing germline genetics into clinical practice}

In 2016, a multi-institutional collaboration reported the finding that over $10 \%$ of men with metastatic prostate cancer have germline 
mutations in DNA repair genes such as BRCA2 and other autosomal dominant high- and medium-penetrance cancer predisposition genes. ${ }^{16}$ This finding led to the practice-changing idea that all men with metastatic prostate cancer be offered genetic testing for germline DNA repair gene mutations. With potential implications for treatment (ie, platinum and PARPi, discussed above) and for family counseling, the intersection of these discoveries now leads to important new challenges and opportunities for prostate cancer clinicians on how best to identify germline mutation carriers. Again, there are important parallels and opportunities when considering what has been learned from the breast and ovarian cancer experience. For example, germline testing of most/all patients is now common in ovarian cancer, which improves selection of systemic treatments and clinical trials that target specific molecular vulnerabilities.

With advances in germline testing for prostate cancer risk genes also comes the opportunity to refine screening for other cancers (eg, pancreas, breast, colon, etc), depending on the specific gene and variant. Cascade germline testing of family members is an important responsibility that can facilitate risk-adapted cancer screening, chemoprevention, reproductive counseling, and research opportunities. Integration of cancer care with genetics services will be increasingly important in research and clinical care for germline cancer risk. Areas of uncertainty that require more collaborative investigation include improved penetrance estimates of individual genes and variants as well as guidelines for management of men with lower penetrance risk genes and variants of uncertain significance. A topic receiving particular attention is the need to diversify populations studied to interpret genetic variants, particularly given that African American men are at increased risk of prostate cancer and most cohorts studied are largely comprised of men of European and/or, in the case of BRCA1/2 mutations, Ashkenazi Jewish ancestry.

Arguably the greatest potential benefit from germline genetic testing may lie in the promise of more refined risk-adapted screening and early detection approaches, which, when combined with appropriate, effective intervention, could avert prostate cancer-specific mortality. Many additional questions remain to be answered, including which patients should undergo genetic testing in the localized disease setting and how management should change. For example, there was lively discussion, but lack of consensus, on how germline mutations should influence decisions about active surveillance, and the potential role for prophylactic prostatectomy in extremely high risk individuals, for example men with clearly pathogenic germline BRCA2 mutations with a family history of death from prostate cancer. Given the rapid evolution in this area, dedicated clinics addressing prostate cancer and genetic risk have been set up to test new models of care and facilitate research efforts at the Seattle Cancer Care Alliance/University of Washington, University of Michigan, Dana-Farber Cancer Institute, Memorial Sloan Kettering Cancer Center, and Sidney Kimmel Cancer Center at Thomas Jefferson University, among others. The consensus from the CHPCA was that further studies are necessary and that close partnership with patients, along with primary care providers, urologists, radiation and medical oncologists, and genetics professionals in these efforts will be critical for success.

\section{3 | Epigenetic regulation of prostate cancer}

Prostate cancer is in large part a disease driven by the androgen receptor (AR), a transcription factor of the steroid nuclear receptor family. ${ }^{17} \mathrm{AR}$ targeting remains a mainstay in the treatment of the disease, both in the first-line with androgen deprivation therapy (ADT), and at metastatic castration-resistance with the potent $A R$ antagonist enzalutamide ${ }^{18}$ and the steroidal CYP17A1 inhibitor abiraterone acetate, which inhibits adrenal production of the natural ligand $5 a$-dihydrotestosterone $(\mathrm{DHT}) .{ }^{19}$ Resistance inevitably develops to these AR-targeting therapies, implying either loss of sensitivity of AR to ligand-dependent inhibition, or a switch in the transcriptional regulation of prostate cancer cells to non-AR-dependent mechanisms. ${ }^{20}$

The transition from normal prostate to cancer is associated with extensive reprogramming of $A R$ binding sites within the genome, also known as the AR cistrome. ${ }^{21}$ In humans, AR cistromes in prostate tumors cluster together and are distinctly different from those in normal prostate tissue. ${ }^{21}$ AR cistrome reprogramming is associated with the transcription factors FOXA1 and HOXB13 in primary prostate tissue $^{21}$ as well as with ERG, a member of the ETS family of transcription factors. ${ }^{22}$ The AR cistrome is distinctly different between normal and primary prostate tumor tissue (M. Pomerantz, et al, unpublished). However, the landscape of the histone acetyl mark $\mathrm{H} 3 \mathrm{~K} 27 \mathrm{Ac}$, an epigenetic marker of active enhancers, is similar between normal and primary prostate tissue but differs in models of metastatic castration-resistant prostate cancer (mCRPC) (M. Pomerantz, et al, unpublished). Integration of histone modification landscapes, including H3K27Ac, with AR and other transcription factor cistromes in models of CRPC, including in the context of enzalutamide and abiraterone resistance, will provide broader insight into epigenetic regulation in advanced prostate cancer.

One such setting is the enzalutamide-resistant prostate cancer xenograft model LREX, derived from LNCaP-AR xenografts selected following prolonged exposure to enzalutamide. ${ }^{23}$ In this model, the glucocorticoid receptor (GR), another member of the steroid nuclear receptor family, is overexpressed and assumes key transcriptional functions of $A R$, driving tumor growth. $G R$ overexpression in tumors has been shown to correlate with poor response to enzalutamide, ${ }^{23}$ and GR activation in the $22 \mathrm{Rv} 1$ prostate cancer model has been shown to promote enzalutamide resistance. ${ }^{24}$ Importantly, GR overexpression in LREX is dynamic and induced by $A R$ inhibition with enzalutamide, with evidence that $A R$ binding to a $G R$ enhancer region suppresses GR expression (N. Shah, et al, unpublished). GR expression also has a role in driving tumor survival and poor outcomes in breast cancer models. ${ }^{25,26}$ These findings offer an opportunity for GR targeting in CRPC with epigenetic modulators that affect GR transcription or with direct GR inhibition. ${ }^{27,28}$ There are likely to be additional epigenetic mechanisms of GR activation amenable to alterative targeting approaches, including dysregulation of glucocorticoid metabolism in tumors through enzalutamide-mediated loss of expression of the metabolic enzyme $11 \beta-H S D 2 .{ }^{29}$

There is clear evidence that epigenetic mechanisms through reprogramming of epigenetic landscapes and use of alternative 
transcription factors including GR are key in prostate cancer progression. It will be critical to define the contexts where these epigenetic driver mechanisms are active in order to exploit them therapeutically, ${ }^{30}$ together with targeting of genomic alterations $s^{6,7,31}$

\section{4 | Histologic transformation to AR-independent aggressive variant prostate cancer}

Approximately a quarter of CRPC patients acquire an "aggressive variant prostate cancer" (AVPC) phenotype, which exhibit low to absent AR levels and expression of neuroendocrine, reprogramming, and stem-related gene signatures. These tumors are indifferent to ARtargeted therapy, and evolve from an adenocarcinoma phenotype. ${ }^{32}$ Recent data suggests that cell reversion to a more plastic state, that is, lineage plasticity, may be driving the emergence of AVPC, and understanding this process is critical to designing therapeutic strategies.

Genomic-sequencing studies and preclinical modeling have highlighted combinatorial loss of function of tumor suppressor genes, PTEN, RB1, and TP53, as key genetic events underlying AVPC progression. $^{32-34}$ Previously, it was demonstrated that genetically engineered mouse models of prostate cancer with over-expression of the large T antigen (TRAMP mice) or co-deletion of TP53 and RB1 develop de novo neuroendocrine prostate cancer (NEPC). ${ }^{35,36}$ Unfortunately, these models do not accurately represent human AVPC and its lineage-plastic phenotype. Recently, mice with a prostate-specific co-deletion of PTEN and RB1 were shown to develop primary and metastatic prostate cancer. ${ }^{37}$ These tumors exhibited co-existence of luminal-like and NEPC-like epithelial cells. Lineage tracing of tumors suggests that AVPC cells evolve from an adenocarcinoma cell. ${ }^{37,38}$ In the PTEN/RB1 double knockout mouse model, a subset of mice whose tumors acquired spontaneous loss-offunction TP53 mutations progressed to castration resistance, indicating that cooperation of TP53, RB1, and PTEN mutations drive evolution of AVPC. ${ }^{37}$ Additionally, double knockout of TP53/RB1 in a human AR dependent prostate cancer cell line (LNCaP-AR) resulted in a lineage plastic phenotype, with phenotypic and gene expression changes $^{39}$ mirroring those described in the PTEN/RB1 double knockout mouse model. In both models, gene expression patterns highly overlapped with published human NEPC gene signatures. ${ }^{40}$

The development of lineage plasticity and castration resistance in prostate cancers deficient in TP53 and RB1 has been found to occur through upregulation of SOX2, a stem cell-associated transcription factor. ${ }^{32,37,39,40}$ SOX2 expression has been found to be regulated by RB1, TP3, and BRN2 (Pou3f2) through various mechanisms (P. Mu, et al. unpublished). ${ }^{41}$

Amplification of N-MYC (MYCN) has also been implicated in driving AVPC progression. ${ }^{40,42,43}$ In a prostate adenocarcinoma mouse model driven by deletion of PTEN, over-expression of N-MYC led to a phenotype shift to poorly differentiated invasive cancer. ${ }^{42}$ This data was in accordance with another study involving combined N-MYC and myristoylated-AKT1 (myrAKT1) over-expression in human prostate cell lines and organoids. ${ }^{43}$ Both studies revealed a gene signature that included positive enrichment of epithelial-to-mesenchymal transition and neuronal/stemness/epigenetic gene signatures, and negative enrichment of AR gene signatures that could distinguish patients with AVPC. ${ }^{42,43}$

Increased expression and amplification of AURKA (Aurora Kinase A), a gene involved in cell cycle, has been observed in human AVPC samples. ${ }^{40}$ These findings led to studies in preclinical AVPC models testing treatment with the allosteric AURKA inhibitor, alisterib, ${ }^{40,42}$ and the initiation of clinical trials testing the AURKA inhibitor MLN8237 in patients with mCRPC and NEPC (NCT01799278; NCT01094288). ${ }^{44}$ Preclinical data also supports further exploration into the treatment of AVPC with CD532, a compound that targets the AURKA:N-MYC interaction and results in degradation of N-MYC. ${ }^{43}$

Over-expression of the epigenetic reprogramming factor $\mathrm{EZH} 2$ is implicated in human and mouse AVPC. ${ }^{32,37,40,42}$ EZH2 inhibition has also been shown to partially reverse AVPC lineage plasticity and resensitize enzalutamide-resistant AVPC models to enzalutamide. ${ }^{37}$ In mouse models, EZH2 can dimerize with N-MYC and AURKA, and $\mathrm{EZH} 2$ inhibition resulted in loss of N-MYC expression. ${ }^{42} \mathrm{~A}$ study using newly generated and characterized human AVPC organoids identified increased expression of EZH2 and its catalytic mark H3K27me3 (L. Puca et al, unpublished). Screening of AVPC organoid lines with a 129chemical compound library in combination with the EZH2 inhibitor GSK503, identified the kinase inhibitors alisertib and neratinib, supporting AURKA and overall kinase inhibition as targets of interest for the treatment of AVPC (L. Puca et al, unpublished). The interaction between N-MYC and EZH2 may explain how chromatin remodeling drives lineage switching and a plastic phenotype. This proposed mechanism is supported by direct interaction between RB1 and EZH2 which directs deposition of $\mathrm{H} 3 \mathrm{~K} 27 \mathrm{me} 3{ }^{45}$ This finding raises a critical question of whether a switch in EZH2 protein interactions drive a molecular switch through altered distribution of $\mathrm{H} 3 \mathrm{~K} 27 \mathrm{me} 3$ in the context of RB1 loss and N-MYC gain of function (L. Ellis and D. Rickman et al, unpublished).

CEACAM5 has also been suggested as a target in AVPC, and studies are underway to test CEACAM5-targeted CAR-T cell immunotherapy (J. Lee et al, unpublished). To date, in vitro data reveal that CEACAM 5 targeting by CAR-T cells show strong on-target toxicity, and preclinical in vivo evaluation is currently underway (J. Lee et al, unpublished).

Overall, these studies highlight that interplay between acquired mutations in tumor suppressor genes and epigenetic and stem cell regulators underlie the transition from an adenocarcinoma to a neuroendocrine or AVPC phenotype in addition to gain of castration resistance. Studies are needed to further clarify the interactions between these factors and identify optimal strategies for treating or preventing AVPC.

\section{5 | Molecular imaging in prostate cancer}

The use of radionuclides (e.g. F18, C11, Zr89) for imaging are redefining how we view disease states in prostate cancer. The posterchild of functional imaging in oncology is positron emission 
tomography (PET) with [F18]-fluorodeoxyglucose (FDG). Unfortunately, due to the low glycolytic activity of prostate cancer, FDG PET plays little to no role in the staging or response assessment for prostate adenocarcinoma. ${ }^{46}$ Therefore, standard of care imaging remains anatomic imaging through CT and monitoring for bone metastasis via technetium bone scans. ${ }^{47}$ The limitations of these modalities are well established, and over the past decade there have been considerable advancements in the development and clinical testing of novel compounds to detect prostate cancer. The primary agents discussed at the CHPCA were C11-choline and PSMA PET.

In the United States, C11-choline has the most evidence to support its use and was approved by the FDA in 2012 for use at the Mayo clinic for recurrent prostate cancer, where over 4000 patients have been scanned to date. For men with recurrent prostate cancer, C11-choline has a reported sensitivity of $95 \%$, specificity of $86 \%$, and is most helpful in men with PSA $>2 \mathrm{ng} / \mathrm{mL}$. ${ }^{48}$ Multiple studies from Mayo clinic have utilized C11-choline to not only document the patterns of relapse after radical prostatectomy and/or salvage radiotherapy, ${ }^{49}$ but to also intervene on these image findings and optimize treatment for recurrent prostate cancer. Utilizing salvage lymph node dissections, often including a retroperitoneal lymph node dissection, the authors have demonstrated the ability to achieve a biochemical response in $79 \%$ of patients, and delay the use of further therapy, such as hormone therapy by $>12$ months in those that recurred. ${ }^{50}$ However, a poorly defined subset of men (21\%) continue to progress and never achieve an undetectable PSA. Randomized trials are utilizing C11-choline to guide oligometastasis directed therapy with a primary endpoint of ADT-free survival, and results from this study are expected in the near future. ${ }^{51}$

Prostate-specific membrane antigen (PSMA) is a transmembrane carboxypeptidase that is expressed in $90-95 \%$ of prostate cancer tumors. There is a direct correlation between expression levels and tumor aggressiveness. ${ }^{52,53}$ PSMA imaging to date has largely focused on documenting the sensitivity and specificity of the radiotracer. The clinical utility and its ability to impact treatment decisions are less established and are the focus of multiple ongoing clinical trials (NCT02981368, NCT02825875). Retrospective comparison studies have demonstrated PSMA PET may outperform F18-choline, can impact salvage radiotherapy treatment planning, and may guide use of stereotactic body radiation therapy (SBRT) for oligometastatic treatment. ${ }^{54-57}$ Critical validation studies are needed. Recently, PSMA PET has been shown to improve initial staging of prostate cancer compared with MRI and provides complimentary information when utilized together. ${ }^{58} \mathrm{~A}$ key challenge of PSMA imaging relates to the numerous small molecules that target PSMA (eg, DKFZ-617, DCFPyL, DCFBC), various radionuclides (F18 and Ga68), and even minibody and full length antibodies that are being tested. Each of these in essence functions as its own "drug" and needs to go through independent testing.

There is little question that these newer molecular imaging modalities are superior at visualizing the location and burden of metastatic disease, but the bar must be set higher to gain regulatory approval and justify the cost of these agents. The consensus from the
CHPCA is that well conducted clinical trials are needed to define the utility, benefit, and value of molecular imaging in prostate cancer. These trials are currently ongoing.

\section{6 | Radionuclide therapy for metastatic castration resistant prostate cancer}

Radionuclides have been used for over a decade to treat prostate cancer. Initial radionuclides, such as beta-emitting strontium-89 and samarium-153, have demonstrated the ability to help palliate painful bone metastasis with no impact on survival. ${ }^{59,60}$ In 2013, radium-223 dichloride, an alpha-emitter, gained FDA approval after the positive phase III ALSYMPCA trial demonstrated not only improvement in pain, but also significant improvements in overall survival. ${ }^{61}$ These exciting results have sparked a resurgence in the interest of utilizing radionuclide therapy to systemically target metastatic prostate cancer.

Because radium-223 can only target bone metastasis, and prostate cancer often harbors disease in lymph node or other visceral sites, new targeting methodologies are being investigated. Given the specificity of PSMA imaging, PSMA-targeting therapeutic agents including small urea-based molecules (eg, PSMA-617) and full length antibodies (eg, J591), conjugated to Lu177 (a beta-emitter) or Ac225 (an alpha-emitter) are being tested. These agents were first tested and reported clinically in German institutions, where multiple retrospective reports demonstrated examples of exceptional responders to both agents, with select cases achieving near complete resolution on molecular imaging and dramatic PSA declines. ${ }^{62-64}$ However, given the retrospective nature of these studies, concerns for toxicity and durability of response, and lack of standardized protocol, prospective trials are now underway around the world, including Australia, Canada, and the United States (notably Weill Cornell [NCT03042468] and UCLA [NCT03042312]). Key questions that remain are how to optimally dose and fractionate these agents, to determine if a small molecule, antibody, or combination of both will be optimal to increase the therapeutic ratio, to determine whether an alpha or beta emitter is optimal with different PSMA-targeting agents, to assess the response rates and long-term impact on progression-free survival (PFS) and overall survival (OS), to quantify the amount and severity of acute and chronic toxicities, and to determine how to further improve outcomes through combinatorial treatment approaches.

\subsection{Optimizing checkpoint immunotherapy for the treatment of prostate cancer}

The immune system is designed to recognize foreign or mutated-self antigens, and as a result, naturally occurring anti-tumor $\mathrm{T}$ cell responses are more frequently observed in tumors with high mutation and high neoantigen loads, such as melanoma, lung cancer, and bladder cancer. These tumor types as well as others with high mutational burden are sensitive to treatment with checkpoint immunotherapy (anti-CTLA4, anti-PD1/PD-L1). Prostate cancer, in contrast, is an "immune desert," typically having a relatively low number of somatic mutations and neoantigens, and hence responses to single agent 
checkpoint immunotherapy have been rare. Exceptions are microsatellite instability (MSI)-high or mismatch repair (MMR)-deficient tumors present in a small subset of mCRPC. 6,16,65,66 The anti-PD-1 antibody, pembrolizumab, recently attained the first-ever cancer-agnostic FDA approval for patients with unresectable MSI-high or MMR-deficient solid tumors, including prostate cancer, ${ }^{67}$ who have progressed on other therapies and have no other satisfactory treatment options. Notably, a study in MSI-high colorectal cancer underscored that the immune status of patients can be a key component of response to checkpoint immunotherapy. ${ }^{68}$ Active work is underway to enhance our understanding of the immune milieu in prostate cancer and to identify the mechanisms that can ignite the immune system against prostate cancer.

Two ongoing clinical trials evaluating checkpoint immunotherapy in prostate cancer have observed improved responses, suggesting possible therapeutic strategies that may benefit a larger fraction of men with prostate cancer. The first is investigating the addition of pembrolizumab after progression on enzalutamide in patients with mCRPC (NCT02312557). ${ }^{69}$ An early interim analysis reported significant PSA responses in $21 \%$ of patients $(n=7 / 34)$ (J. Graff et al., unpublished). ${ }^{69}$ An expansion cohort of 30 patients has been added to this study for a total of 58 patients. A second ongoing trial is testing the combination of the PARP inhibitor olaparib with the anti-PD-L1 therapy durvalumab in heavily pre-treated $\mathrm{mCPRC}$ patients unselected for any genomic alteration (NCT02484404) (F. Karzai et al, unpublished). ${ }^{70}$ PSA declines have been observed in $68 \%$ of the 19 evaluable patients and partial responses in 4 of 12 evaluable patients thus far. This study has been expanded to include another 40 patients for a total of 65. Expansion cohorts in both trials will provide opportunities to better understand the mechanisms of action and patients most likely to benefit. Since the reporting of these two trials, several other studies testing similar therapeutic strategies are being initiated.

The role for targeting immune checkpoints beyond CTLA- 4 and PD1/PD-L1 is being explored in prostate and other cancers. VISTA is an immune checkpoint molecule recently found to be upregulated in prostate cancer. ${ }^{71}$ A VISTA-targeting therapy is currently being tested in a phase I clinical trial (NCT02671955), and if shown to be safe, may lead to trials in prostate cancer.

Alternatively, agonist antibodies activating co-stimulatory molecules expressed on T cells may be an opportunity for rousing antitumor T cells. One potential target is OX40, a T cell costimulatory molecule that promotes $\mathrm{T}$ cell expansion, differentiation, and survival. Combining OX40-activating antibodies with CTLA4-blockade has been reported to be synergistic in inducing anti-tumor CD8 T cells, eliciting tumor regression, and extending survival in prostate mouse models. ${ }^{72}$ Anti-OX40/anti-CTLA4 treatment in these mice was found to elicit a population of CD8 T cells expressing high levels of EOMES, a marker of central memory T cells. EOMES-high CD8 T cells were characterized by increased proliferation rates and lower levels of checkpoint molecules (PD1, LAG3), compared with EOMES-low CD8 T cells, suggesting these cells may be resistant to negative regulatory effects of checkpoint molecules (W. Redmond, et al, unpublished). Finally, anti-tumor T cell responses elicited by anti-OX40/anti-CTLA4 treatment may be further enhanced by addition of a vaccine or radiation therapy, ${ }^{73}$ (W. Redmond, et al, unpublished).

\subsection{Rationale for combining radiation therapy with immunotherapy}

Radiation therapy can modulate the immune system through release of antigens, induction of tumor cell necrosis, activation and expansion of effector and helper T cells, upregulation of MHC-1, and chemokine release. ${ }^{74,75}$ There is data to suggest CD8+ T cells may be required for tumor killing by radiotherapy. ${ }^{76}$ Immune stimulation may also contribute to the therapeutic effects of radium-223. Exposure to sublethal doses of radium-223 has been shown to increase the susceptibility of prostate, lung and breast cancer cell lines to $T$ cell mediated killing. ${ }^{77}$ In this study, radium-223 treatment was associated with the induction of CD8+ cytotoxic T cells specific to the tumor antigens CEA, MUC-1 and brachyury, and was accompanied by increased expression of $\mathrm{MHC}-1$ and calreticulin, which are proteins integral to effective antigen presentation. ${ }^{77}$

Based on these and other observations, many studies have begun exploring the activity of combining radiation therapy with immunotherapy for the treatment of prostate cancer. Radiation therapy has been shown to influence the $T$ cell repertoire when given in combination with checkpoint blockade. ${ }^{78}$ In vivo models found increases in PD-1/PD-L1 expression on immune and tumor cells after radiation and significantly enhanced efficacy with the addition of anti-PD-L1 to radiation. ${ }^{79}$ Those same studies demonstrated decreased levels of immunosuppressive tumoral myeloid derived suppressor cells (MDSCs), which were mediated through tumor necrosis factor (TNF). ${ }^{79}$ A reduction of PD-1 expression on circulating CD8+ and CD4+ T cells has been observed after a single treatment of radium- $223 .{ }^{80}$ Radium-223 is a rational partner for immune stimulating agents such as PD-1 pathway blockade and Sipuleucel-T due to its efficacy, tolerability, and potential for favorable immunomodulation. Multiple trials are ongoing or planned to test the addition of radium-223 to immunotherapies such as pembrolizumab (NCT03093428), atezolizumab (NCT02814669), and Sipuleucel-T (NCT02463799).

\section{9 | Tumor vaccine strategies for prostate cancer}

Vaccines that target tumor-associated antigens are being explored in the clinic as an approach to eliciting anti-tumor immune responses in tumor types that have a paucity of neoantigens. Approximately 75 vaccine platforms are being explored in prostate cancer clinical trials, mostly in phase I or II settings. Prostate cancer vaccines that have made it to phase III testing include Sipuleucel-T, GVAX, Prostvac, ProstAtak, and DCVAC/PCa. Sipuleucel-T is an autologous cellular vaccine in which antigen presenting cells are immunized ex vivo against the tumor associated antigen prostatic acid phosphatase (PAP) in the presence of the immune stimulatory cytokine GM-CSF. Sipuleucel-T was FDA-approved based on a 4.1 month improvement in median overall survival over placebo in $\mathrm{mCRPC} .{ }^{81}$ Prostvac is the next furthest along in development and is currently being tested in the phase III 
PROSPECT trial (NCT01322490). Prostvac is a virus-based vaccine encoding PSA, along with immune costimulatory molecules LFA-3, ICAM-1, and B7-1. Results from PROSPECT are anticipated by the end of 2017. If positive, Prostvac may join Sipuleucel-T as an FDAapproved vaccine for prostate cancer.

Vaccines alone are unlikely to induce dramatic long-term tumor regression in prostate cancer, and combinations are being explored with various other types of immunotherapy including checkpoint blockade. An ongoing trial at the $\mathrm{NCl}$ is testing the combination of Prostvac with ipilimumab and/or nivolumab (NCT02933255). This trial includes correlative studies to examine the impact of the different treatment combinations on the immune compartment in the tumor microenvironment.

Plasmid-based DNA vaccine strategies have been employed to induce tumor antigen-specific Th1-based T cell responses and have the advantage of being highly modifiable to improve antigen targeting. Plasmid-based DNA vaccines targeting the highly immunogenic protein SSX-2, a protein involved in stem cell migration and metastatic potential and which is expressed on approximately $25 \%$ of prostate cancer metastases (pTVG-SSX2), have demonstrated induction of robust Th1 immune responses. ${ }^{82,83}$ Combining pTVG-SSX2 with PD-1 blockade resulted in enhanced antitumor effects and immune responses both in mouse models and in patients enrolled on a phase I clinical trial, suggesting that combination therapy may be more effective. ${ }^{84,85}$ A phase II study is underway combining the pTVG-SSX2 vaccine with PD-1 blockade in metastatic prostate cancer (NCT02499835).

\subsection{0 | Priming the immune system}

The optimal timing of immunotherapy is not well defined; however, rationale exists for earlier administration during the disease course when the immune system may be more intact and the disease burden is low. Neoadjuvant immunotherapy prior to surgery for clinically localized disease has the potential to downstage tumors, eliminate micrometastatic disease, and generate durable memory $\mathrm{T}$ cell responses that prevent or delay recurrences. Additionally, the ability to examine surgical tissue provides the opportunity to investigate immunomodulatory mechanisms.

GM-CSF has been explored as an immune adjuvant in patients with prostate cancer with the goals of inducing both innate and adaptive immunity by activating antigen presenting cells with the subsequent induction of effector antitumor $T$ cell responses. GM-CSF may also promote an anti-tumor M1-like phenotype in macrophages, resulting in direct tumor killing and stimulation of a specific T-cell mediated anti-tumor response. ${ }^{86-88}$ In a 13 patient study of GM-CSF in the metastatic castration-resistant setting, the majority of patients (92\%) exhibited some degree of PSA decline, and one patient had a sustained and deep PSA response with associated improvement in bone scans for over 14 months. ${ }^{89}$

In a neoadjuvant study, the effects of 2-4 weeks of GM-CSF prior to surgery $(n=18)$ was explored, using prostatectomy specimens from untreated subjects as matched controls. ${ }^{90}$ While GM-CSF was well tolerated and modulated PSA with the majority experiencing some degree of PSA decline, only $11 \%$ of patients had a $>50 \%$ PSA decline. ${ }^{90}$ Induction of a transient peripheral immune response was evidenced by proliferation of cytotoxic and helper $T$ cells and inhibitory $T$ regulatory cells (Tregs). ${ }^{90}$ Significant dose-dependent increases in effector and helper $\mathrm{T}$ cell infiltration at the tumor interface and in the tumor were also observed. ${ }^{90}$ However, when compared with clinical responses to PD-1 blockade observed in melanoma, ${ }^{91}$ the level of immune infiltration induced by GM-CSF in prostate cancer suggests limited benefit as a monotherapy, though it may have a role as an adjuvant in combination strategies. Indeed, the maturing phase III Prostvac study is poised to address the question of whether adding GM-CSF to Prostvac enhances efficacy (NCT01322490).

\subsection{1 | Rationale for combining androgen deprivation therapy with immunotherapy}

Evidence suggests that standard therapies such as ADT or radiation may already be immunomodulatory. ${ }^{74,92}$ Androgen deprivation may stimulate lymphocyte and B-cell development, potentiate immune responses to vaccines, revive thymopoiesis, and abrogate tolerance to prostate cancer specific antigens. ${ }^{93}$ Robust $\mathrm{T}$ cell infiltration has been observed in the prostate after androgen ablation, and appears to be composed more of CD4+ than CD8+ T cells. ${ }^{94}$ A MycCap model of prostate cancer demonstrated that castration resulted in an initial $\mathrm{T}$ cell rich pro-inflammatory and suppressive infiltrate, which evolves as the tumor progresses to castration-resistance (Y. Chen and C. Drake, et al, submitted).

In mice, castration was found to synergize with depletion of Tregs with an anti-CTLA-4 antibody, resulting in improved control of tumor growth (T. Nirschl and C. Drake, et al, submitted). This concept was tested in a neoadjuvant study of Degarelix alone or in combination with the GM-CSF vaccine GVAX and the Treg-depleting agent cyclophosphamide, ${ }^{95}$ followed by prostatectomy, in 32 men with high risk prostate cancer (NCT01696877). In both study arms, immune stimulation was evidenced by increased CD8 infiltration into tumors following treatment, however this was rivaled by an equivalent increase in Treg infiltration (C. Drake, et al, submitted). Nevertheless, the addition of GVAX + cyclophosphamide to Degaralix resulted in numerically improved time to PSA recurrence and time to next treatment (C. Drake, et al, submitted). Overall, these human and murine models highlight the complexity of the immune milieu in prostate cancer and suggest that adding immunomodulatory agents such as Treg depleting agents to standard hormone therapy may be a fruitful strategy.

\subsection{Targeting other immune cells in the tumor microenvironment}

Targeting immune-suppressive cells such as myeloid cells and Tregs in the tumor microenvironment may be promising strategies to enhance anti-tumor $\mathrm{T}$ cell responses and improve clinical outcomes. B cells can also antagonize anti-tumor $T$ cell activity. In a recent study, mouse 
models of prostate cancer were found to be unresponsive to low dose oxaliplatin. ${ }^{96}$ However, anti-tumor $\mathrm{T}$ cell responses were observed if $\mathrm{B}$ cells were depleted, suggesting that low-dose oxaliplatin is immunogenic and supporting a tumor-promoting role for $\mathrm{B}$ cells. ${ }^{96}$ In these models, anti-PD-L1 also synergized with low-dose oxaliplatin in blocking tumor growth. ${ }^{96}$ These studies support further exploration into the role of $\mathrm{B}$ cells in prostate cancer progression, treatment resistance, and as a therapeutic target.

Natural Killer (NK) cells are an innate immune cell type with potent tumor killing potential. NK cells act by targeting cells expressing ligands to activating NK receptors. These ligands, such as MIC (MHC I Chain related Molecule), are induced by cellular stresses and implicate cells as being potentially infected or cancerous. ${ }^{97}$ MIC is highly expressed by prostate cancer cells as an early response to DNA damage or oxidative stress. ${ }^{97,98}$ However, a soluble form of MIC (sMIC) can be produced by advanced prostate cancer cells, and acts to block the activity of NK cells, impair T cell function, and expand immune suppressive myeloid cells. ${ }^{98-}$ ${ }^{100}$ A neutralizing sMIC antibody (mAb B10G5) has been developed and was demonstrated to reduce primary tumor burden and eliminate metastases in mouse models of prostate cancer. ${ }^{101} \mathrm{~B} 10 \mathrm{G} 5$ was found to synergize with anti-CTLA-4 therapy, ${ }^{102}$ suggesting that improved antitumor immunity may be achieved by simultaneous activation of $\mathrm{T}$ cells and NK cells. Efforts are underway to complete the preclinical studies necessary to prepare B10G5 for testing in prostate cancer clinical trials.

\section{I CONCLUSION}

The 2017 CHPCA Meeting was a highly productive and interactive experience, with over 400 questions posed across 34 talks. Topics with the greatest immediate and near-term impact included: (i) how to screen for and differentially treat men with germline DNA repair gene mutations; (ii) understanding and optimizing immunotherapy combinations for prostate cancer; and (iii) optimizing PSMA radionuclide therapy for prostate cancer. PCF will transform these gaps in knowledge to action through funded research. One immediate outcome of the meeting was the formation of a PCF-led working group on DNA repair, which will address questions related to germline genetics, optimizing methods to identify germline and somatic mutations, and understanding the biology of various genetic alterations and their association with treatment responses and resistance to PARPi and platinum chemotherapy.

The theme of the 2018 CHPCA Meeting will be: "Tumor Cell Heterogeneity and Resistance."

\section{DISCLOSURES}

LCH: Advisory: Bayer, Genentech, Dendreon, Pfizer, Medivation/ Astellas, Kew Group, Theragene, Corvus, Merck; Research to the institution: Bayer, Sotio, Bristol-Myers Squibb, Merck, Takeda, Dendreon/Valient, Janssen, Medivation/Astellas, Genentech, Pfizer; CME: PER. DES: One time advisory board for Dendreon. None of the other authors declare any potential conflicts of interest.

\section{ORCID}

Andrea K. Miyahira (iD http://orcid.org/0000-0003-4976-002X

Lauren C. Harshman (iD http://orcid.org/0000-0002-7636-1588

\section{REFERENCES}

1. Pienta KJ, Walia G, Simons JW, Soule HR. Beyond the androgen receptor: new approaches to treating metastatic prostate cancer. Report of the 2013 Prouts Neck Prostate Cancer Meeting. Prostate. 2014;74:314-320.

2. Miyahira AK, Kissick HT, Bishop JL, et al. Beyond immune checkpoint blockade: new approaches to targeting host-tumor interactions in prostate cancer: report from the 2014 Coffey-Holden prostate cancer academy meeting. Prostate. 2015;75:337-347.

3. Miyahira AK, Lang JM, Den RB, et al. Multidisciplinary intervention of early, lethal metastatic prostate cancer: report from the 2015 Coffey-Holden Prostate Cancer Academy Meeting. Prostate. 2016; 76:125-139.

4. Miyahira AK, Roychowdhury S, Goswami S, et al. Beyond seed and soil: understanding and targeting metastatic prostate cancer; report From the 2016 Coffey-Holden Prostate Cancer Academy Meeting. Prostate. 2017;77:123-144.

5. Keller ET, Rowley DR, Tomlins SA, et al. Eleventh prouts neck meeting on prostate cancer: emerging strategies in prostate cancer therapy. Cancer Res. 2007;67:9613-9615.

6. Robinson D, Van Allen EM, Wu YM, et al. Integrative clinical genomics of advanced prostate cancer. Cell. 2015;161: 1215-1228.

7. Mateo J, Carreira S, Sandhu S, et al. DNA-repair defects and olaparib in metastatic prostate cancer. N Engl J Med. 2015;373:1697-1708.

8. Cheng HH, Pritchard CC, Boyd T, Nelson PS, Montgomery B. Biallelic Inactivation of BRCA2 in Platinum-sensitive metastatic castrationresistant prostate cancer. Eur Urol. 2015.

9. Sakai W, Swisher EM, Karlan BY, et al. Secondary mutations as a mechanism of cisplatin resistance in BRCA2-mutated cancers. Nature. 2008;451:1116-1120.

10. Swisher EM, Sakai W, Karlan BY, Wurz K, Urban N, Taniguchi T. Secondary BRCA1 mutations in BRCA1-mutated ovarian carcinomas with platinum resistance. Cancer Res. 2008;68:2581-2586.

11. Norquist B, Wurz KA, Pennil CC, et al. Secondary somatic mutations restoring $B R C A 1 / 2$ predict chemotherapy resistance in hereditary ovarian carcinomas. J Clin Oncol. 2011;29:3008-3015.

12. Barber $\amalg$, Sandhu S, Chen L, et al. Secondary mutations in BRCA2 associated with clinical resistance to a PARP inhibitor. J Pathol. 2013;229:422-429.

13. Quigley D, Alumkal JJ, Wyatt AW, et al. Analysis of circulating cellfree DNA identifies multi-clonal heterogeneity of BRCA2 reversion mutations associated with resistance to PARP inhibitors. Cancer Discov. 2017.

14. Goodall J, Mateo J, Yuan W, et al. Circulating free DNA to guide prostate cancer treatment with PARP inhibition. Cancer Discov. 2017.

15. Cancer Genome Atlas Research, N. The molecular taxonomy of primary prostate cancer. Cell. 2015;163:1011-1025.

16. Pritchard CC, Mateo J, Walsh MF, et al. Inherited DNA-Repair gene mutations in men with metastatic prostate cancer. N Engl J Med. 2016;375:443-453.

17. Heinlein CA, Chang C. Androgen receptor in prostate cancer. Endocr Rev. 2004;25:276-308.

18. Tran C, Ouk S, Clegg NJ, et al. Development of a second-generation antiandrogen for treatment of advanced prostate cancer. Science. 2009;324:787-790. 
19. de Bono JS, Logothetis CJ, Molina A, et al. Abiraterone and increased survival in metastatic prostate cancer. N Engl J Med. 2011;364: 1995-2005.

20. Watson PA, Arora VK, Sawyers CL. Emerging mechanisms of resistance to androgen receptor inhibitors in prostate cancer. Nat Rev Cancer. 2015;15:701-711.

21. Pomerantz MM, Li F, Takeda DY, et al. The androgen receptor cistrome is extensively reprogrammed in human prostate tumorigenesis. Nat Genet. 2015;47:1346-1351.

22. Chen $Y$, Chi P, Rockowitz $S$, et al. ETS factors reprogram the androgen receptor cistrome and prime prostate tumorigenesis in response to PTEN loss. Nat Med. 2013;19:1023-1029.

23. Arora VK, Schenkein E, Murali R, et al. Glucocorticoid receptor confers resistance to antiandrogens by bypassing androgen receptor blockade. Cell. 2013;155:1309-1322.

24. Isikbay M, Otto K, Kregel S, et al. Glucocorticoid receptor activity contributes to resistance to androgen-targeted therapy in prostate cancer. Horm Cancer. 2014;5:72-89.

25. Moran TJ, Gray S, Mikosz CA, Conzen SD. The glucocorticoid receptor mediates a survival signal in human mammary epithelial cells. Cancer Res. 2000;60:867-872.

26. Pan D, Kocherginsky M, Conzen SD. Activation of the glucocorticoid receptor is associated with poor prognosis in estrogen receptornegative breast cancer. Cancer Res. 2011;71:6360-6370.

27. Asangani IA, Wilder-Romans K, Dommeti VL, et al. BET bromodomain inhibitors enhance efficacy and disrupt resistance to AR antagonists in the treatment of prostate cancer. Mol Cancer Res. 2016;14:324-331.

28. Kach J, Long TM, Selman P, et al. Selective glucocorticoid receptor modulators (SGRMs) delay castrate-resistant prostate cancer growth. Mol Cancer Ther. 2017.

29. Li J, Alyamani M, Zhang A, et al. Aberrant corticosteroid metabolism in tumor cells enables $\mathrm{GR}$ takeover in enzalutamide resistant prostate cancer. Elife. 2017;6.

30. Yegnasubramanian S. Prostate cancer epigenetics and its clinical implications. Asian J Androl. 2016;18:549-558.

31. Abida W, Armenia J, Gopalan A, et al. Prospective genomic profiling of prostate cancer across disease states reveals germline and somatic alterations that may affect clinical decision making. JCO Precision Oncol. 2017;1-16.

32. Beltran H, Prandi D, Mosquera JM, et al. Divergent clonal evolution of castration-resistant neuroendocrine prostate cancer. Nat Med. 2016;22:298-305.

33. Tan HL, Sood A, Rahimi HA, et al. Rb loss is characteristic of prostatic small cell neuroendocrine carcinoma. Clin Cancer Res. 2014;20: 890-903.

34. Aparicio AM, Shen L, Tapia EL, et al. Combined tumor suppressor defects characterize clinically defined aggressive variant prostate cancers. Clin Cancer Res. 2016;22:1520-1530.

35. Gingrich JR, Greenberg NM. A transgenic mouse prostate cancer model. Toxicol Pathol. 1996;24:502-504.

36. Zhou Z, Flesken-Nikitin A, Corney DC, et al. Synergy of p53 and Rb deficiency in a conditional mouse model for metastatic prostate cancer. Cancer Res. 2006;66:7889-7898.

37. Ku SY, Rosario S, Wang Y, et al. Rb1 and Trp53 cooperate to suppress prostate cancer lineage plasticity, metastasis, and antiandrogen resistance. Science. 2017;355:78-83.

38. Zou M, Toivanen R, Mitrofanova A, et al. Transdifferentiation as a mechanism of treatment resistance in a mouse model of castrationresistant prostate cancer. Cancer Discov. 2017;7:736-749.

39. Mu P, Zhang Z, Benelli M, et al. SOX2 promotes lineage plasticity and antiandrogen resistance in TP53- and RB1-deficient prostate cancer. Science. 2017;355:84-88.

40. Beltran H, Rickman DS, Park K, et al. Molecular characterization of neuroendocrine prostate cancer and identification of new drug targets. Cancer Discov. 2011:1:487-495.
41. Bishop JL, Thaper D, Vahid S, et al. The master neural transcription factor BRN2 is an androgen receptor-suppressed driver of neuroendocrine differentiation in prostate cancer. Cancer Discov. 2017;7: 54-71.

42. Dardenne E, Beltran H, Benelli M, et al. Induces an EZH2-Mediated transcriptional program driving neuroendocrine prostate cancer. Cancer Cell. 2016;30:563-577.

43. Lee JK, Phillips JW, Smith BA, et al. N-Myc drives neuroendocrine prostate cancer initiated from human prostate epithelial cells. Cancer Cell. 2016;29:536-547.

44. Lin J, Patel SA, Sama AR, et al. A phase I/II study of the investigational drug alisertib in combination with abiraterone and prednisone for patients with metastatic castration-resistant prostate cancer progressing on abiraterone. Oncologist. 2016;21:1296-1297e.

45. Ishak CA, Marshall AE, Passos DT, et al. An RB-EZH2 complex mediates silencing of repetitive DNA sequences. Mol Cell. 2016;64: 1074-1087.

46. Jadvar H. PET of glucose metabolism and cellular proliferation in prostate cancer. J Nucl Med. 2016;57:25S-29S.

47. Mohler JL, Armstrong AJ, Bahnson RR, et al. Prostate cancer, version 1.2016. J Natl Compr Canc Netw. 2016;14:19-30.

48. Mitchell CR, Lowe VJ, Rangel LJ, Hung JC, Kwon ED, Karnes RJ. Operational characteristics of (11)c-choline positron emission tomography/computerized tomography for prostate cancer with biochemical recurrence after initial treatment. J Urol. 2013;189: 1308-1313.

49. Parker WP, Evans JD, Stish BJ, et al. Patterns of recurrence after postprostatectomy fossa radiation therapy identified by $\mathrm{C}-11$ choline positron emission Tomography/Computed tomography. Int J Radiat Oncol Biol Phys. 2017;97:526-535.

50. Zattoni F, Nehra A, Murphy CR, et al. Mid-term outcomes following salvage lymph node dissection for prostate cancer nodal recurrence status Post-radical prostatectomy. Eur Urol Focus. 2:522-531.

51. Decaestecker K, De Meerleer G, Ameye F, et al. Surveillance or metastasis-directed therapy for Oligometastatic prostate cancer recurrence (STOMP): study protocol for a randomized phase II trial. BMC Cancer. 2014;14:671.

52. Ross JS, Sheehan CE, Fisher HA, et al. Correlation of primary tumor prostate-specific membrane antigen expression with disease recurrence in prostate cancer. Clin Cancer Res. 2003;9:6357-6362.

53. Barinka C, Rojas C, Slusher B, Pomper M. Glutamate carboxypeptidase II in diagnosis and treatment of neurologic disorders and prostate cancer. Curr Med Chem. 2012;19:856-870.

54. Afshar-Oromieh A, Zechmann CM, Malcher A, et al. Comparison of PET imaging with a (68)Ga-labelled PSMA ligand and (18)F-cholinebased PET/CT for the diagnosis of recurrent prostate cancer. Eur $\mathrm{J}$ Nucl Med Mol Imaging. 2014;41:11-20.

55. Henkenberens $C$, von Klot CA, Ross TL, et al. (68)Ga-PSMA ligand $\mathrm{PET} / \mathrm{CT}$-based radiotherapy in locally recurrent and recurrent oligometastatic prostate cancer: Early efficacy after primary therapy. Strahlenther Onkol. 2016;192:431-439.

56. van Leeuwen PJ, Stricker P, Hruby G, et al. (68) Ga-PSMA has a high detection rate of prostate cancer recurrence outside the prostatic fossa in patients being considered for salvage radiation treatment. BJU Int. 2016;117:732-739.

57. Bluemel C, Linke F, Herrmann K, et al. Impact of 68Ga-PSMA PET/CT on salvage radiotherapy planning in patients with prostate cancer and persisting PSA values or biochemical relapse after prostatectomy. EJNMMI Res. 2016;6:78

58. Eiber M, Weirich G, Holzapfel K, et al. Simultaneous 68Ga-PSMA HBED-CC PET/MRI improves the localization of primary prostate cancer. Eur Urol. 2016;70:829-836.

59. Porter AT, McEwan AJ, Powe JE, et al. Results of a randomized phase-III trial to evaluate the efficacy of strontium-89 adjuvant to local field external beam irradiation in the management of endocrine 
resistant metastatic prostate cancer. Int J Radiat Oncol Biol Phys. 1993;25:805-813.

60. Sartor O, Reid RH, Hoskin PJ, et al. Samarium-153-Lexidronam complex for treatment of painful bone metastases in hormonerefractory prostate cancer. Urology. 2004;63:940-945.

61. Parker C, Nilsson S, Heinrich D, et al. Alpha emitter radium-223 and survival in metastatic prostate cancer. N Engl J Med. 2013;369: 213-223.

62. Kratochwil C, Giesel FL, Stefanova M, et al. PSMA-Targeted radionuclide therapy of metastatic castration-Resistant prostate cancer with 177Lu-Labeled PSMA-617. J Nucl Med. 2016;57: 1170-1176.

63. Fendler WP, Reinhardt S, Illhan $\mathrm{H}$, et al. Preliminary experience with dosimetry, response and patient reported outcome after 177LuPSMA-617 therapy for metastatic castration-resistant prostate cancer. Oncotarget. 2017;8:3581-3590.

64. Kratochwil C, Bruchertseifer F, Giesel FL, et al. 225Ac-PSMA-617 for PSMA-targeted alpha-radiation therapy of metastatic castrationResistant prostate cancer. J Nucl Med. 2016;57:1941-1944.

65. Schweizer MT, Cheng HH, Tretiakova MS, et al. Mismatch repair deficiency may be common in ductal adenocarcinoma of the prostate. Oncotarget. 2016;7:82504-82510.

66. Miyahira AK, Soule HR. The 23 rd annual prostate cancer foundation scientific retreat report. Prostate. 2017;77:1093-1106.

67. Le DT, Durham JN, Smith KN, et al. Mismatch-repair deficiency predicts response of solid tumors to PD-1 blockade. Science. 2017.

68. Mlecnik B, Bindea G, Angell HK, et al. Integrative analyses of colorectal cancer show immunoscore is a stronger predictor of patient survival than microsatellite instability. Immunity. 2016;44: 698-711.

69. Graff JN, Alumkal JJ, Drake CG, et al. Early evidence of anti-PD-1 activity in enzalutamide-resistant prostate cancer. Oncotarget. 2016;7:52810-52817.

70. Karzai F, Madan RA, Owens H, et al. Combination of PDL-1 and PARP inhibition in an unselected population with metastatic castrateresistant prostate cancer (mCRPC). J Clin Oncol. 2017;35: 5026-5026.

71. Gao J, Ward JF, Pettaway CA, et al. VISTA is an inhibitory immune checkpoint that is increased after ipilimumab therapy in patients with prostate cancer. Nat Med. 2017;23:551-555.

72. Redmond WL, Linch SN, Kasiewicz MJ. Combined targeting of costimulatory (OX40) and coinhibitory (CTLA-4) pathways elicits potent effector $\mathrm{T}$ cells capable of driving robust antitumor immunity. Cancer Immunol Res. 2014;2:142-153.

73. Linch SN, Kasiewicz MJ, McNamara MJ, Hilgart-Martiszus IF, Farhad M, Redmond WL. Combination OX40 agonism/CTLA-4 blockade with HER2 vaccination reverses T-cell anergy and promotes survival in tumor-bearing mice. Proc Natl Acad Sci USA. 2016;113: E319-E327.

74. Hodge JW, Guha C, Neefjes J, Gulley JL. Synergizing radiation therapy and immunotherapy for curing incurable cancers. Opportunities and challenges. Oncology (Williston Park). 2008;22: 1064-1070. Discussion 1075, 1080-1, 1084.

75. Takeshima T, Chamoto K, Wakita D, et al. Local radiation therapy inhibits tumor growth through the generation of tumor-specific CTL: its potentiation by combination with Th1 cell therapy. Cancer Res. 2010;70:2697-2706.

76. Lee Y, Auh SL, Wang Y, et al. Therapeutic effects of ablative radiation on local tumor require $\mathrm{CD} 8+\mathrm{T}$ cells: changing strategies for cancer treatment. Blood. 2009;114:589-595.

77. Malamas AS, Gameiro SR, Knudson KM, Hodge JW. Sublethal exposure to alpha radiation (223Ra dichloride) enhances various carcinomas' sensitivity to lysis by antigen-specific cytotoxic $T$ lymphocytes through calreticulin-mediated immunogenic modulation. Oncotarget. 2016;7:86937-86947.
78. Twyman-Saint Victor C, Rech AJ, Maity A, et al. Radiation and dual checkpoint blockade activate non-redundant immune mechanisms in cancer. Nature. 2015;520:373-377.

79. Deng L, Liang H, Burnette B, et al. Irradiation and anti-PD-L1 treatment synergistically promote antitumor immunity in mice. J Clin Invest. 2014;124:687-695.

80. Kim JW, Shin KY, Deshpande M, et al. Changes in T cell immunity in patients with metastatic castration resistant prostate treated with Radium-223 treatment. J Clin Oncol. 2016;34.

81. Kantoff PW, Higano CS, Shore ND, et al. Sipuleucel-T immunotherapy for castration-resistant prostate cancer. New Eng J Med. 2010;363:411-422.

82. Smith HA, Cronk RJ, Lang JM, McNeel DG. Expression and immunotherapeutic targeting of the SSX family of cancer-testis antigens in prostate cancer. Cancer Res. 2011;71:6785-6795.

83. Smith HA, Rekoske BT, McNeel DG. DNA vaccines encoding altered peptide ligands for SSX2 enhance epitope-specific CD8+ T-cell immune responses. Vaccine. 2014;32:1707-1715.

84. Rekoske BT, Olson BM, McNeel DG. Antitumor vaccination of prostate cancer patients elicits PD-1/PD-L1 regulated antigen-specific immune responses. Oncoimmunology. 2016;5: e1165377.

85. Rekoske BT, Smith HA, Olson BM, Maricque BB, McNeel DG. PD-1 or PD-L1 blockade restores antitumor efficacy following SSX2 epitope-Modified DNA vaccine immunization. Cancer Immunol Res. 2015;3:946-955.

86. Fleetwood AJ, Lawrence T, Hamilton JA, Cook AD. Granulocytemacrophage colony-stimulating factor (CSF) and macrophage CSFdependent macrophage phenotypes display differences in cytokine profiles and transcription factor activities: implications for CSF blockade in inflammation. J Immunol. 2007;178:5245-5252.

87. Hamilton JA. Colony-stimulating factors in inflammation and autoimmunity. Nat Rev Immunol. 2008;8:533-544.

88. Verreck FA, de Boer T, Langenberg DM, et al. Human IL-23producing type 1 macrophages promote but IL-10-producing type 2 macrophages subvert immunity to (myco)bacteria. Proc Natl Acad Sci USA. 2004;101:4560-4565.

89. Small EJ, Reese DM, Um B, Whisenant S, Dixon SC, Figg WD. Therapy of advanced prostate cancer with granulocyte macrophage colony-stimulating factor. Clin Cancer Res. 1999;5:1738-1744.

90. Wei XX, Chan S, Kwek S, et al. Systemic GM-CSF recruits effector $t$ cells into the tumor microenvironment in localized prostate cancer. Cancer Immunol Res. 2016;4:948-958.

91. Tumeh PC, Harview CL, Yearley JH, et al. PD-1 blockade induces responses by inhibiting adaptive immune resistance. Nature. 2014;515:568-571.

92. Nesslinger NJ, Sahota RA, Stone B, et al. Standard treatments induce antigen-specific immune responses in prostate cancer. Clin Cancer Res. 2007;13:1493-1502.

93. Aragon-Ching JB, Williams KM, Gulley JL. Impact of androgendeprivation therapy on the immune system: implications for combination therapy of prostate cancer. Front Biosci. 2007;12: 4957-4971.

94. Mercader M, Bodner BK, Moser MT, et al. T cell infiltration of the prostate induced by androgen withdrawal in patients with prostate cancer. Proc Natl Acad Sci USA. 2001;98:14565-14570.

95. Wada S, Yoshimura K, Hipkiss EL, et al. Cyclophosphamide augments antitumor immunity: studies in an autochthonous prostate cancer model. Cancer Res. 2009;69:4309-4318.

96. Shalapour S, Font-Burgada J, Di Caro G, et al. Immunosuppressive plasma cells impede T-cell-dependent immunogenic chemotherapy. Nature. 2015;521:94-98.

97. Wu JD, Lin DW, Page ST, Lundgren AD, True LD, Plymate SR. Oxidative DNA damage in the prostate may predispose men to a higher risk of prostate cancer. Transl Oncol. 2009;2:39-45. 
98. Liu G, Lu S, Wang X, et al. Perturbation of NK cell peripheral homeostasis accelerates prostate carcinoma metastasis. J Clin Invest. 2013;123:4410-4422.

99. Wu JD, Higgins LM, Steinle A, Cosman D, Haugk K, Plymate SR. Prevalent expression of the immunostimulatory MHC class I chainrelated molecule is counteracted by shedding in prostate cancer. J Clin Invest. 2004;114:560-568.

100. Xiao G, Wang X, Sheng J, Lu S, Yu X, Wu JD. Soluble NKG2D ligand promotes MDSC expansion and skews macrophage to the alternatively activated phenotype. J Hematol Oncol. 2015;8:13.

101. Lu S, Zhang J, Liu D, et al. Nonblocking monoclonal antibody targeting soluble MIC revamps endogenous innate and adaptive antitumor responses and eliminates primary and metastatic tumors. Clin Cancer Res. 2015;21:4819-4830.
102. Zhang J, Liu D, Li G, et al. Antibody-mediated neutralization of soluble MIC significantly enhances CTLA4 blockade therapy. Sci Adv. 2017;3:e1602133.

How to cite this article: Miyahira AK, Cheng HH, Abida W, et al. Beyond the androgen receptor II: New approaches to understanding and treating metastatic prostate cancer; Report from the 2017 Coffey-Holden Prostate Cancer Academy Meeting. The Prostate. 2017;77:1478-1488. https://doi.org/10.1002/pros.23424 